\title{
Search for MSSM Higgs bosons and beyond at CMS
}

\section{Chayanit Asawatangtrakuldee ${ }^{* \dagger}$}

Deutsches Elektronen-Synchrotron (DESY), Hamburg

E-mail: chayanitecern.ch

Searches for beyond the Standard Model (BSM) Higgs bosons are presented, particularly in the context of MSSM and Higgs Triplet Model, including a neutral Higgs boson decays into two tau leptons, charged Higgs bosons in decays of tau lepton and neutrino, charged Higgs bosons in decays of vector bosons, and doubly-charged Higgs boson in three and four lepton final states. The data is collected with the CMS detector at the LHC which corresponds to integrated luminosities of 2.3 and $12.9 \mathrm{fb}^{-1}$ at center-of-mass energy of $13 \mathrm{TeV}$ in 2015 and 2016, respectively. No signature of BSM Higgs boson is observed. The upper limits are placed on the cross-section times branching fraction for each search, as well as interpreted in different models of an extended Higgs sector.

EPS-HEP 2017, European Physical Society conference on High Energy Physics 5-12 July 2017

Venice, Italy

*Speaker.

${ }^{\dagger}$ on behalf of the CMS Collaboration 


\section{Introduction}

The discovery of the Higgs boson at $125 \mathrm{GeV}$ and the studies of its properties by the ATLAS and CMS [1] collaborations [2, 3, 4] at the CERN LHC have shown a compatibility with the Standard Model (SM) Higgs boson and, hence, led to a new era in physics. However, the SM is known not to be complete, and a number of BSM theories include an extended Higgs sector. As such, studies of any potential deviations from the SM predictions in the properties of the existing Higgs boson, together with searches for possible additional Higgs bosons, are essential in the hunt for new physics.

There exist many possible extensions of the SM, and one of the simplest is the Minimal Supersymmetric Standard Model (MSSM), which incorporates supersymmetry. In the MSSM, two scalar Higgs doublets are introduced and the symmetry is spontaneously broken twice giving three neutral, $\phi=\mathrm{h}, \mathrm{H}$, A and two charged, $\mathrm{H}^{+}$and $\mathrm{H}^{-}$, Higgs bosons. Of the neutral Higgs states, the $\mathrm{h}$ and $\mathrm{H}$ are $\mathrm{CP}$-even, while the $\mathrm{A}$ is $\mathrm{CP}$-odd. At tree level, only two parameters, conventionally chosen to be the mass of the pseudoscalar Higgs, $m_{\mathrm{A}}$, and the ratio of the two vacuum expectation values (vevs), $\tan \beta=v_{1} / v_{2}$, are needed to define the properties of the Higgs sector in the MSSM.

For a large number of MSSM models which have small and medium values of $\tan \beta$, the dominant production mode is gluon fusion as in the SM. The existence of the second Higgs doublet means that for large values of $\tan \beta$ couplings to down-type fermions are enhanced. This leads to two effects: enhanced rate of decays into taus, and a second dominant production mode, $b$ associated production. In this report, the first results of direct searches for MSSM Higgs bosons decaying into a pair of tau leptons from the CMS collaboration using the data from 2016 running are presented.

Charged Higgs bosons appear in many extensions of the SM with at least two Higgs doublets or even more complicated Higgs sectors. In this report, searches for charged Higgs bosons in decays of $\mathrm{H}^{ \pm} \rightarrow \tau^{ \pm} v_{\tau}$ and $\mathrm{H}^{ \pm} \rightarrow \mathrm{WZ}$ are also presented. However, couplings of charged Higgs bosons to vector bosons are strongly suppressed in the MSSM but can appear in Higgs sectors extended by SU(2) triplets, particularly the Georgi-Machacek (GM) model where real and complex Higgs triplets with vevs are arranged to preserve custodial symmetry. In such models, the charged Higgs bosons are produced via vector boson fusion (VBF) and the couplings depend on $\mathrm{m}\left(\mathrm{H}^{ \pm}\right)$and the parameter $\sin \theta_{\mathrm{H}}$.

In addition, recent experiments have discovered the phenomenon of neutrino flavour oscillations, which is only possible if neutrino has non-zero mass. One possibility to introduce neutrino masses is a so-called the minimal Type-II seesaw mechanism, as part of Higgs Triplet Model. Measuring the branching fractions of the doubly-charged Higgs boson would give access to neutrino parameters such as masses. A search for a doubly-charged Higgs boson, $\Phi^{ \pm \pm}$, considering three lepton and four lepton final states is also introduced in this report.

Detailed studies on each search are given in next sections.

\section{Search for a neutral MSSM Higgs boson decaying into $\tau \tau$}

The direct searches for MSSM Higgs bosons decaying into a pair of tau leptons are performed in four most sensitive final states of the taus: $\mathrm{e} \mu, \mathrm{e} \tau_{h}, \mu \tau_{h}$ and $\tau_{h} \tau_{h}$, where $\tau_{h}$ indicates a tau 
decaying hadronically [5]. The dataset corresponds to an integrated luminosity of $12.9 \mathrm{fb}^{-1}$ at center-of-mass energy of $13 \mathrm{TeV}$. Events are categorised depending on the presence of a b tagged jet in the event. The $b$-tag category consists of events with at least one $b$ tagged jet in order to select events from $b$ associated production, and events in the no $b$-tag category have exactly zero $b$ tagged jets to cover gluon fusion production. Background from irreducible $\mathrm{Z} / \gamma^{*} \rightarrow \tau \tau$ is estimated from simulation with correction derived from data. Other backgrounds from DY+jets, W+jets, QCD and $\mathrm{t} \overline{\mathrm{t}}$ are estimated from either data in control regions or simulations depending on channels.

The total transverse mass is the final discriminating variable used to search for an excess due to signal. The binned distributions of total transverse mass are fitted simultaneously in all four channels and the b-tag and no b-tag categories, using a binned maximum likelihood fit. No evidence for a signal has been found. Upper limits are set at 95\% confidence level (CL) on the cross-section times branching fraction for the two dominant production modes, gluon fusion ( $\mathrm{gg} \phi$ ) and $b$-associated production $(b b \phi)$. The limits are set assuming a single resonance signal with narrow width. Figure 1 shows these limits as a function of Higgs boson mass, $m_{\phi}$.
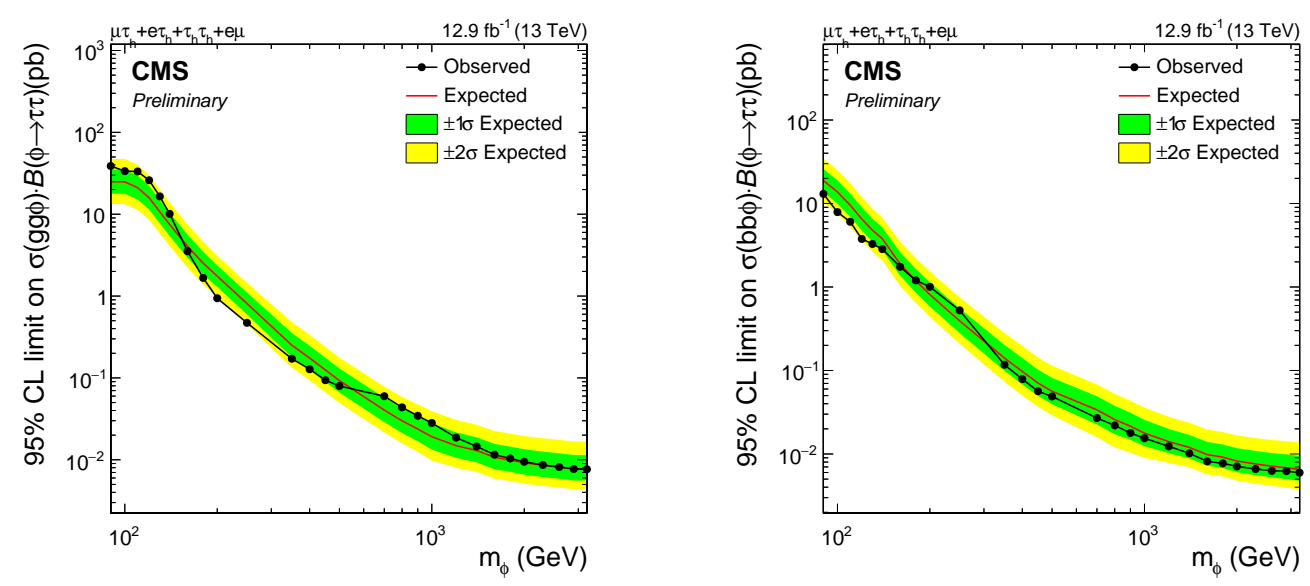

Figure 1: Expected and observed limits on cross-section times branching fraction for (left) the gluon fusion process $(\mathrm{gg} \phi)$ and (right) the b-associated production process $(\mathrm{bb} \phi)$, resulting from the combination of all four channels [5].

Limits are also set on $m_{\mathrm{A}}$ and $\tan \beta$ in two different benchmark models; the $m_{\mathrm{h}}^{\bmod +}$ and hMSSM scenarios $[6,7]$. The results are shown in Figure 2.

\section{Search for charged Higgs bosons with the $\mathrm{H}^{ \pm} \rightarrow \tau^{ \pm} v_{\tau}$}

A search for charged Higgs bosons in decays of $\mathrm{H}^{ \pm} \rightarrow \tau^{ \pm} v_{\tau}$ is performed both with mass larger than the top quark in the $\mathrm{pp} \rightarrow \mathrm{H}^{ \pm}$tb production, and lighter than the top quark in the $\mathrm{pp} \rightarrow$ $\mathrm{H}^{ \pm} \mathrm{W}^{\mp}$ bb channel [8]. The fully hadronic final state is considered in order to ensure neutrinos only originated from a charged Higgs boson, and thus the transverse mass variable $\left(m_{\mathrm{T}}\right)$ can be reconstructed and performed signal extraction using a binned maximum likelihood fit.

The observation agrees with the SM prediction. Model-independent upper limits on the branching fraction of $B\left(\mathrm{t} \rightarrow \mathrm{bH}^{ \pm}\right) \times B\left(H^{ \pm} \rightarrow \tau^{ \pm} v_{\tau}\right)$ at $95 \% \mathrm{CL}$ are set for the mass range between 

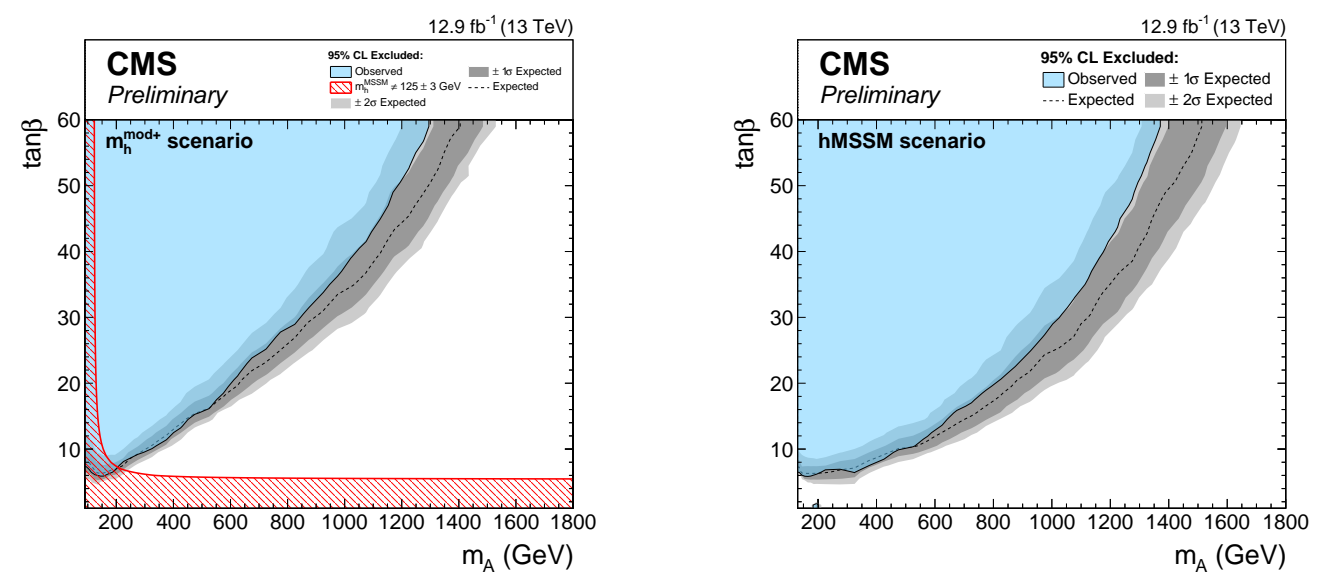

Figure 2: Model dependent exclusion limits in the $m_{\mathrm{A}}-\tan \beta$ plane, combining all channels, for the $m_{\mathrm{h}}^{\bmod +}$ (left) and hMSSM (right) scenarios. In left plot, the red contour indicates the region which does not yield a Higgs boson consistent with a mass of $125 \mathrm{GeV}$ within the theoretical uncertainties of $\pm 3 \mathrm{GeV}$ [5].

80-160 GeV and shown on Figure 3 (left), while upper limits on the production cross-section $\sigma\left(\mathrm{pp} \rightarrow \mathrm{H}^{ \pm} \mathrm{tb}\right)$ times the branching fraction $B\left(H^{ \pm} \rightarrow \tau^{ \pm} v_{\tau}\right)$ are presented on Figure 3 (right) for masses in the range $180 \mathrm{GeV}$ to $3 \mathrm{TeV}$. Moreover, limits are interpreted in the context of the $m_{\mathrm{h}}^{\text {mod}}+$ benchmark scenario as shown in Figure 4.
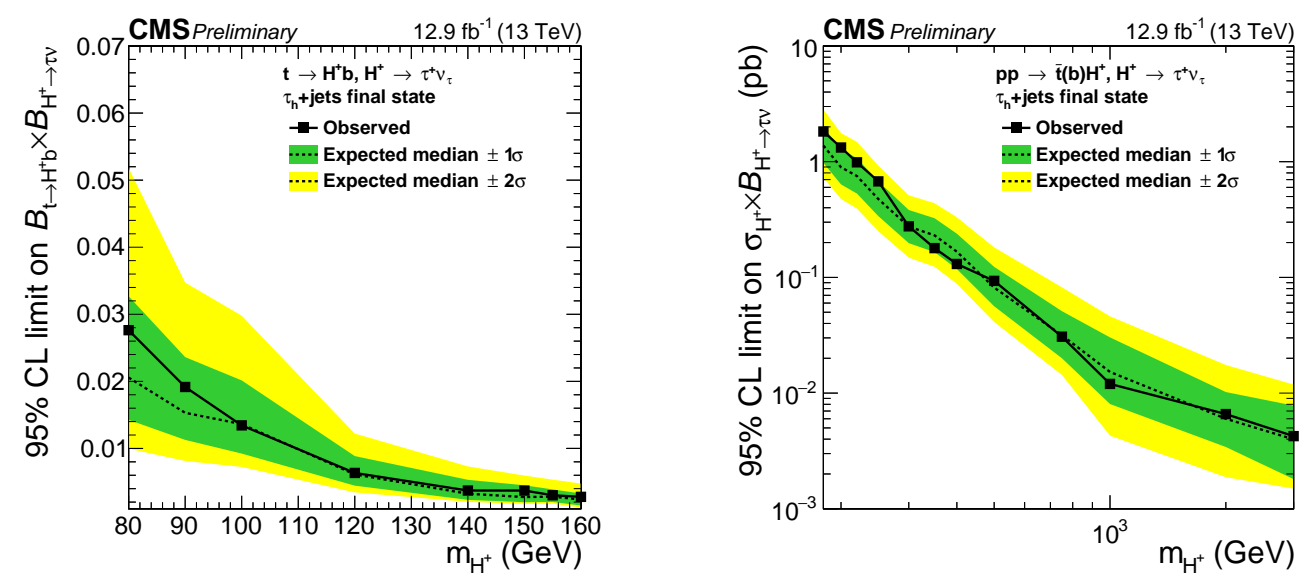

Figure 3: The observed 95\% CL exclusion limits (solid points) on $B\left(\mathrm{t} \rightarrow \mathrm{bH} \mathrm{H}^{ \pm}\right) \times B\left(H^{ \pm} \rightarrow \tau^{ \pm} v_{\tau}\right)$ (left) and $\sigma\left(\mathrm{pp} \rightarrow \mathrm{H}^{ \pm} \mathrm{tb}\right) \cdot B\left(H^{ \pm} \rightarrow \tau^{ \pm} v_{\tau}\right)$ (right) is compared to the expectations from the SM model (dashed line). The green (yellow) error bands represent one (two) standard deviations of the expected limit [8].

\section{Search for charged Higgs bosons with the $\mathrm{H}^{ \pm} \rightarrow \mathrm{WZ}$}

A search for charged Higgs bosons produced via vector boson fusion and decaying into $\mathrm{W}$ and $\mathrm{Z}$ bosons is performed using 2015 and 2016 data with integrated luminosity of $15.2 \mathrm{fb}^{-1}$ [9]. The event selection requires three leptons (electrons or muons) from $\mathrm{W}$ and $\mathrm{Z}$ boson decays, two jets 

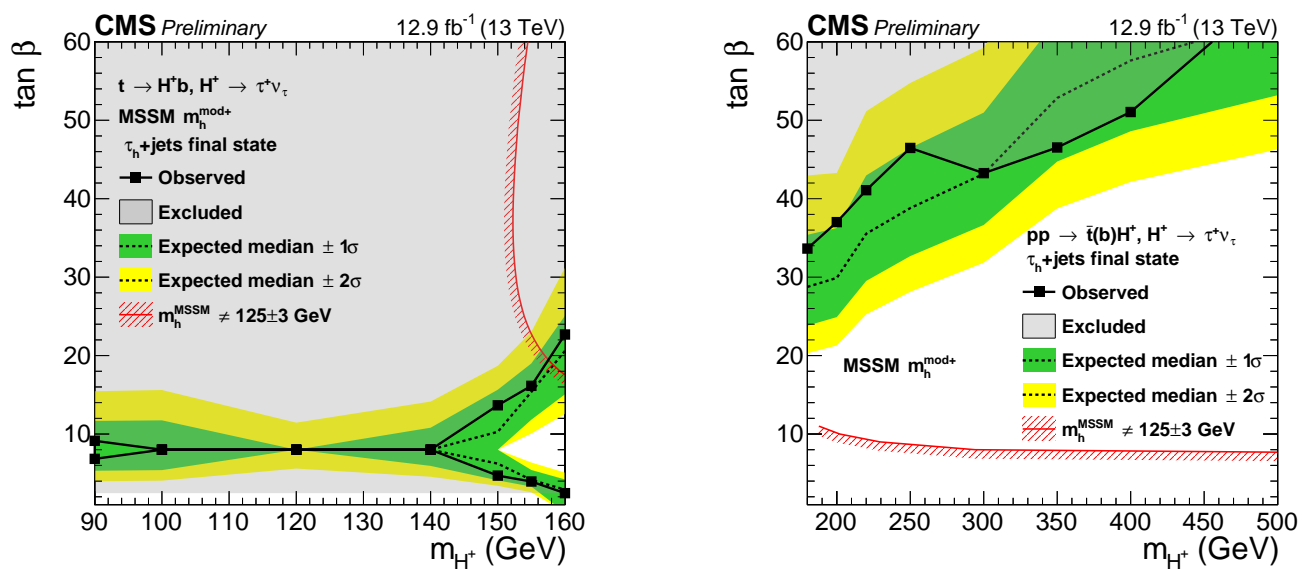

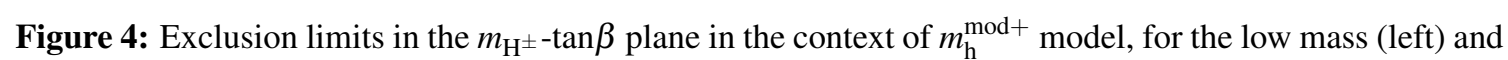
the high mass (right) searches [8].

with large pseudo-rapidity separation, high dijet mass, and missing transverse momentum in VBF topology. The data are compared to the predictions of the GM model for a charged Higgs boson mass range of $200<m\left(\mathrm{H}^{ \pm}\right)<1000 \mathrm{GeV}$.

After applying the full selection, 9 and 62 events are selected in the data collected in 2015 and 2016, respectively. They agree with the SM predictions and no excess due to a possible signal is observed. Model-independent exclusion limits are set on $\sigma_{\mathrm{VBF}}\left(\mathrm{H}^{ \pm}\right) \times B\left(\mathrm{H}^{ \pm} \rightarrow \mathrm{WZ}\right)$ at $95 \%$ $\mathrm{CL}$ and shown in Figure 5 (left). These exclusion limits are also compared to the predicted crosssections at NNLO in the GM model in the $s_{\mathrm{H}^{-}}-m\left(\mathrm{H}^{ \pm}\right)$plane as shown in Figure 5 (right).
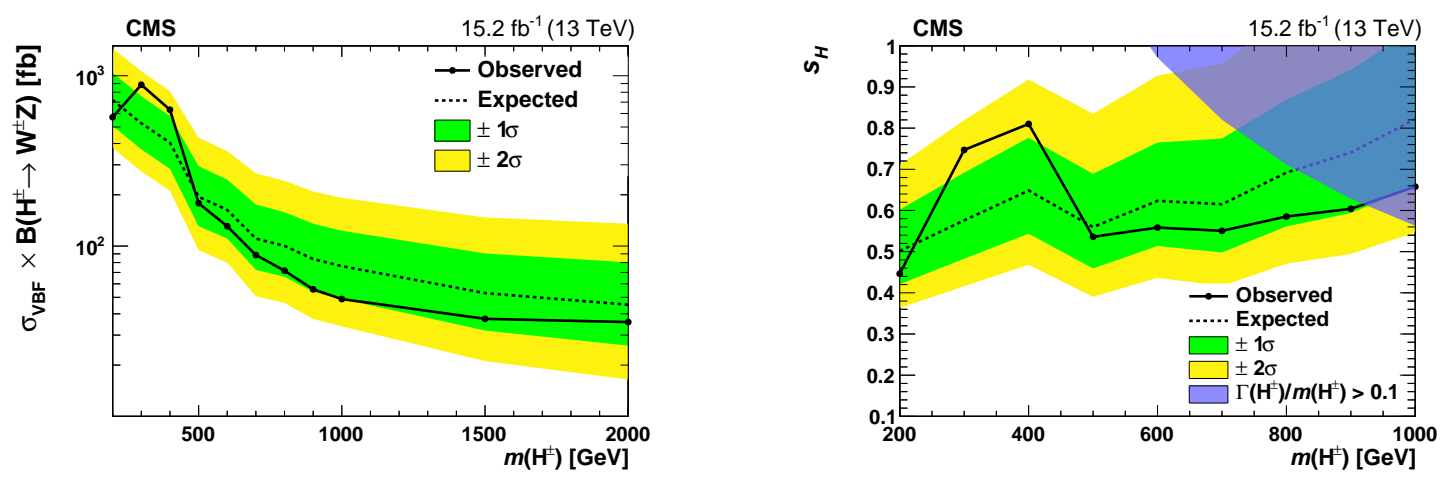

Figure 5: Expected and observed exclusion limits at $95 \%$ confidence level as a function of $m\left(\mathrm{H}^{ \pm}\right)$for $\sigma_{\mathrm{VBF}}\left(\mathrm{H}^{ \pm}\right) \times B\left(\mathrm{H}^{ \pm} \rightarrow \mathrm{WZ}\right)$ (left) and on the ratio of vacuum expectation values in the GM model (right). The blue shaded area covers the theoretically not allowed parameter space [9].

\section{Search for doubly-charged Higgs boson production in 3 and 4 lepton final states}

A search for a doubly-charged Higgs boson, $\Phi^{ \pm \pm}$, is performed using 2016 data [10], including final states with three leptons coming from the associated production of $\Phi^{ \pm \pm} \Phi^{\mp}$ and four leptons 
coming from the pair production of $\Phi^{++} \Phi^{--}$. This analysis is well motivated by the minimal Type-II seesaw mechanism in which the branching fractions are not fixed by the model and a model independent search can be performed.

No significant excess over the SM background expectation is observed, and lower bounds on the doubly-charged Higgs boson mass are derived for a variety of assumptions on its branching ratio to charged lepton pairs, as shown in Figure 6.

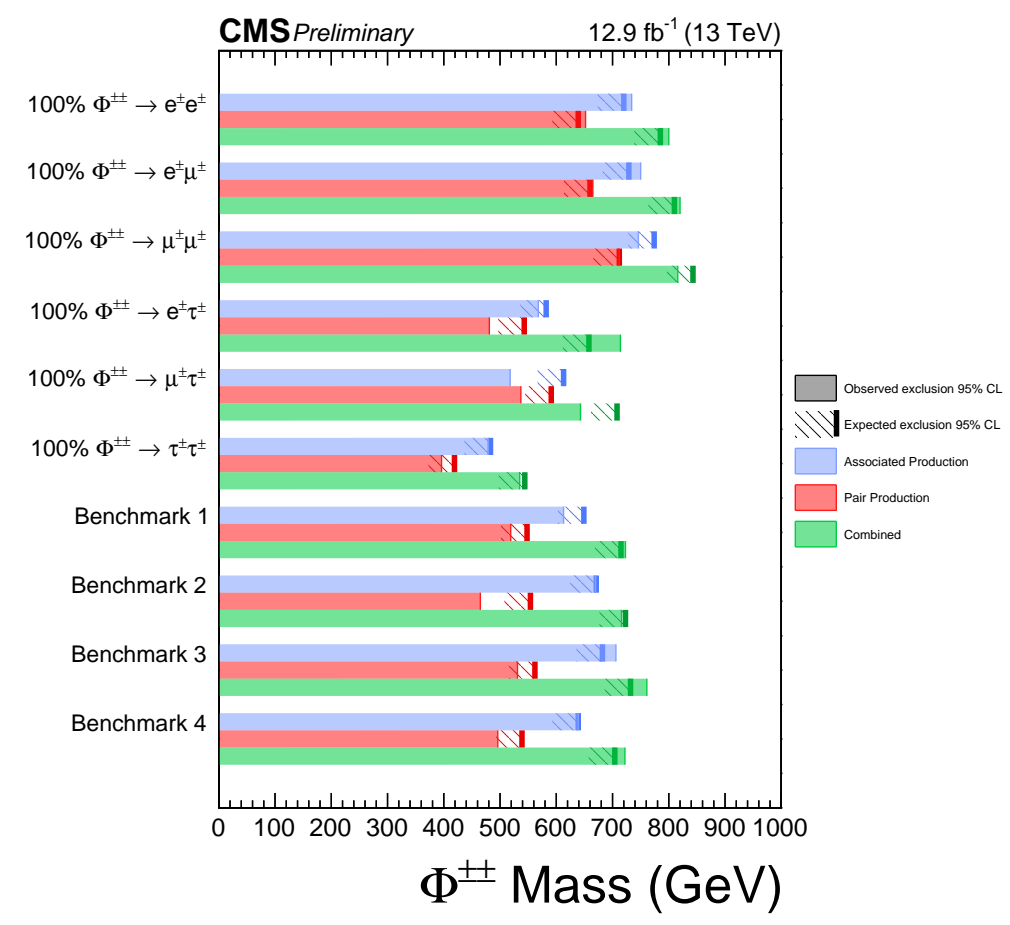

Figure 6: Summary of expected and observed limits for each production mode and the combined limit. The shaded region represents the excluded mass points and the thick solid line represents the expected exclusion with the hashed region indicating the direction [10].

\section{Summary}

Several searches for BSM Higgs boson have been carried out in the CMS experiment using 2015 and 2016 data at center-of-mass energy of $13 \mathrm{TeV}$. No evidence of additional Higgs bosons has been observed. The upper limits are provided on the cross-section times branching fraction for different searches. The results are, furthermore, interpreted in the context of an extended Higgs sector, particularly MSSM and Higgs Triplet Model.

All the presented searches are being continued with the full 2016 Run-II data, in which the integrated luminosity has already reached $36 \mathrm{fb}^{-1}$. This amount of integrated luminosity will give a substantial boost to the sensitivity for new physics phenomena of the Higgs boson.

\section{References}

[1] CMS Collaboration, The CMS experiment at the CERN LHC, JINST 3 S08004 (2008). 
[2] ATLAS Collaboration, Observation of a new particle in the search for the Standard Model Higgs boson with the ATLAS detector at the LHC, Phys. Lett. B 716 (2012) 1.

[3] CMS Collaboration, Observation of a new boson at a mass of $125 \mathrm{GeV}$ with the CMS experiment at the LHC, Phys. Lett. B 716 (2012) 30.

[4] CMS Collaboration, Observation of a new boson with mass near $125 \mathrm{GeV}$ in pp collisions at $\sqrt{s}=7$ and $8 \mathrm{TeV}$, JHEP 06 (2013) 081.

[5] CMS Collaboration, Search for a neutral MSSM Higgs boson decaying into $\tau \tau$ with $12.9 \mathrm{fb}^{-1}$ of data at $\sqrt{s}=13 \mathrm{TeV}$, CMS-PAS-HIG-16-037, http: / / cds. cern. ch/record/2231507.

[6] M. Carena et al., MSSM Higgs Boson Searches at the LHC: Benchmark Scenarios after the Discovery of a Higgs-like Particle, Eur. Phys. J. C. 73 (2013), no. 9, 2552.

[7] E. Bagnaschi et al., Benchmark scenarios for low tan $b$ in the MSSM, Technical Report LHCHXSWG-2015-002, CERN, Geneva, Aug, 2015.

[8] CMS Collaboration, Search for charged Higgs bosons with the $\mathrm{H}^{ \pm} \rightarrow \tau^{ \pm} v_{\tau}$ decay channel in the fully hadronic final state at $\sqrt{s}=13 \mathrm{TeV}$, CMS-PAS-HIG-16-031, http: //cds.cern. ch/record/2223865.

[9] CMS Collaboration, Search for charged Higgs bosons produced via vector boson fusion and decaying into a pair of $W$ and $Z$ bosons using proton-proton collisions at $\sqrt{s}=13 \mathrm{TeV}$, CMS-PAS-HIG-16-027, arXiv:1705.02942.

[10] CMS Collaboration, A search for doubly-charged Higgs boson production in three and four lepton final states at $\sqrt{s}=13 \mathrm{TeV}$, CMS-PAS-HIG-16-036, http://cds.cern. ch/record/2242956. 